\title{
Uso de analgésicos antiinflamatorios no esteroidales en ortodoncia: Revisión bibliográfica
}

\author{
Use of Nonsteroidal Anti-inflammatory Drugs in Orthodontics: Literature review
}

\author{
María Catalina Álvarez-Parker', Daniela Andrea Rojas-Cáceres², Katerín Nicol Terán-Quezada
}

Para citar este artículo: Álvarez-Parker MC, Rojas-Cáceres DA, Terán-Quezada KN. Uso de analgésicos antiinflamatorios no esteroidales en ortodoncia: Revisión bibliográfica. Ustasalud 2018; 17: 48-56

Licencia Creative Commons

\section{(c) (1) (\$) $\odot$} lo tanto, los lectores pueden acceder libremente a los artículos en su formato .pdf, igualmente podrán descargarlos y difundirlos; sin embargo no podrán modificarlos o alterarlos, adicionalmente se debe reconocer la autoría de las personas que figuran en las publicaciones, pero estas no podrán ser comercializadas.

\begin{abstract}
${ }^{1}$ Cirujano dentista, licenciada en Odontología, UFT, Práctica Pública-Privada.

${ }^{2}$ Cirujano dentista, licenciada en Odontología, UAndes, Práctica Privada.

${ }^{3}$ Cirujano dentista, licenciada en Odontología, CAS-UDD, Práctica Pública-Privada.

Autor de correspondencia: María Catalina Álvarez Parker Correo electrónico: dra.calvarezp@gmail.com
\end{abstract}

\section{RESUMEN}

Un $90 \%$ de los pacientes con tratamiento de ortodoncia relatan presentar dolor postoperatorio a la aplicación de fuerzas mecánicas, alcanzando el máximo disconfort a las 24 horas. Estudios sugieren como método de elección el uso de analgésicos antiinflamatorios no esteroidales (AINES) para el control y manejo del dolor.

El objetivo de la presente revisión fue evaluar literatura existente sobre AINES utilizados en pacientes sometidos a tratamientos ortodóncicos y su relación con el proceso inflamatorio, revisar alternativas farmacológicas y sugerir el medicamento más apropiado para el manejo y control del dolor.

Para llevar a cabo este estudio se utilizaron bases de datos como Pubmed, Scielo, ScienceDirect, EBSCOhost, y Google Académico, teniendo como límites de búsqueda documentos publicados entre los años 2006 y 2018, a excepción de dos estudios, uno del año 1996 y otro del 2000, los cuáles proporcionaron información de suma relevancia. Se consideraron artículos como revisiones sistemáticas, estudios prospectivos y retrospectivos.

Se concluyó que es importante manejar el dolor involucrado en los tratamientos ortodóncicos sin interferir en el proceso inflamatorio que lo acompaña. Al analizar diferentes AINES no se observaron diferencias significativas en cuanto a la reducción del dolor, a excepción del Paracetamol, el cual se considera el más apropiado, debido a que este concentra su acción a nivel del SNC, no alterando la síntesis de prostaglandinas y permitiendo el movimiento dental. Se sugiere que se hagan estudios sobre medicamentos como Indometacina, Diclofenaco, Flurbiprofeno y Etoricoxib, debido a que existe escaza evidencia al respecto.

Palabras clave: Dolor ortodóncico, movimiento dental, ortodoncia, inflamación, AINES.

\section{ABSTRACT}

$90 \%$ of patients with orthodontic treatment report postoperative pain to the application of mechanical forces, reaching maximum discomfort at 24 hours. Studies suggest the use of non-steroidal anti-inflammatory analgesics (NSAIDs) for the control and management of pain as the method of choice.

The aim of the present review was to evaluate existing literature on NSAIDs used in patients undergoing orthodontic treatment and its relationship with the inflammatory process, review pharmacological alternatives and suggest the most appropriate medication for the management and control of pain.

In order to carry out this study, databases such as Pubmed, Scielo, ScienceDirect, EBSCOhost, and Google Academic were used, having as search limits documents published between 2006 and 2018, with the exception of two studies, one from 1996 and the other from 2000, which provided information of great relevance. Articles were considered as systematic reviews, prospective and retrospective studies.

It was concluded that it is important to manage the pain involved in orthodontic treatments without interfering with the inflammatory process that accompanies it. When analyzing different NSAIDs, no significant differences were observed in terms of pain reduction, with the exception of Paracetamol, which is considered the most appropriate, because it concentrates its action at the CNS level, not altering the synthesis of Prostaglandins and allowing the dental movement. It is suggested that studies be conducted on drugs such as Indomethacin, Diclofenac, Flurbiprofen and Etoricoxib, due to the lack of evidence in this regard.

Keywords: Orthodontic pain, dental movement, orthodontics, inflammation, NSAIDs. 


\section{INTRODUCCIÓN}

A lo largo de los años, la literatura ha intentado definir y explicar los tratamientos ortodóncicos investigando el movimiento dentario y su complejidad ${ }^{1}$. Existen diversas teorías al respecto, una de ellas, la teoría de presión-tensión explica el movimiento a través de tres etapas: alteraciones en el flujo sanguíneo debido a la reducción o aumento del diámetro de los vasos sanguíneos asociados a la presión existente en el ligamento periodontal; respuestas bioquímicas relacionadas a la formación o liberación de mediadores y mensajeros químicos provenientes de tejidos de soporte $y$, por último, activación celular que involucra presencia de osteoclastos y osteoblastos ${ }^{1,2}$.

Es por esto que, sin importar cuán avanzado esté el tratamiento o cuán competente sea el profesional, el tratamiento ortodóncico siempre está asociado a disconfort. Específicamente, entre un 90 a un $95 \%$ de los pacientes relatan presentar molestias y dolor posterior a la aplicación de fuerzas ${ }^{1,3}$. Este malestar se expresa como una sensación de presión, tensión, dolor dental y dolor general en la cavidad oral ${ }^{4}$. Es por lo anterior, que se debe tener en cuenta que mientras mayor sea el dolor asociado, menor será la adhesión al tratamiento del paciente ${ }^{1}$.

Fisiológicamente el dolor se clasifica como una respuesta nociceptiva, producida por la estimulación de terminaciones nerviosas libres ${ }^{5}$. Posterior a este estímulo, se produce la liberación de moléculas proinflamatorias, estimulando las vías aferentes de fibras nerviosas A-delta y fibras C, llevando el impulso nervioso ${ }^{6}$. Se debe tener en consideración la presencia del dolor como una experiencia sensorial y emocional desagradable, asociada a una injuria de naturaleza multifactorial ${ }^{7}$.

A pesar de los intentos realizados, aún no se ha definido un estándar de atención para controlar el dolor causado por la ortodoncia. A través de los años, se han propuesto varios métodos como, la administración de analgésicos antiinflamatorios no esteroidales (AINES), el uso de láser de baja intensidad, estimulación vibratoria del ligamento periodontal, estimulación nerviosa eléctrica transcutánea, entre otros ${ }^{7,8}$.

El uso de analgésicos es la modalidad comúnmente utilizada para controlar el dolor asociado con el tra- tamiento de ortodoncia ${ }^{8-12}$. Los AINES bloquean la síntesis de prostaglandinas a partir del ácido araquidónico, mediante la inhibición de la enzima ciclooxigenasa, además, se ha identificado que las prostaglandinas tienen una acción directa en el aumento del número y tamaño de osteoclastos, y en la estimulación de su actividad reabsortiva ${ }^{13,14}$. Es por lo anterior que, en los últimos años, el uso de AINES en el control del dolor en ortodoncia ha sido cuestionado. Porque, no solo tendría un efecto positivo en cuanto a analgesia, sino que también podría afectar la secuencia del movimiento dentario, mediante la inhibición o disminución de la relación entre la inflamación y el proceso de reabsorción ósea, disminuyendo el rango de movimiento dentario $\log \operatorname{rado}^{14,15}$.

El objetivo de la presente revisión fue evaluar la literatura existente sobre los analgésicos antiinflamatorios no esteroidales (AINES) utilizados en pacientes sometidos a tratamientos ortodóncicos y su relación con el proceso inflamatorio, revisar alternativas farmacológicas y sugerir el medicamento más apropiado para el manejo y control del dolor.

\section{MATERIALES Y MÉTODOS}

Se realizó una revisión de la literatura científica, a través de la búsqueda de artículos en las siguientes bases de datos: Pubmed, Scielo, ScienceDirect, EBSCOhost, y Google Académico. Al realizar esta búsqueda se utilizaron palabras claves como: dolor ortodóncico, movimiento dental, ortodoncia, inflamación, AINES, analgesia.

Con el fin de seleccionar los artículos analizados, se utilizaron como criterios de inclusión, artículos que debían contener las palabras de búsqueda (dolor ortodóncico, movimiento dental, ortodoncia, inflamación, AINES) tener acceso a texto completo, ser publicaciones en español e inglés, y limitarse a los últimos 12 años, a excepción de un estudio de 1996 y uno del 2000 , los cuales proporcionaron información importante sobre el manejo del dolor (Figura 1.).

Para definir el nivel de evidencia científica y grado de recomendación, se utilizó el esquema propuesto por la Agencia de Evaluación de Tecnología Médica $(\mathrm{AATM})^{16}$. 


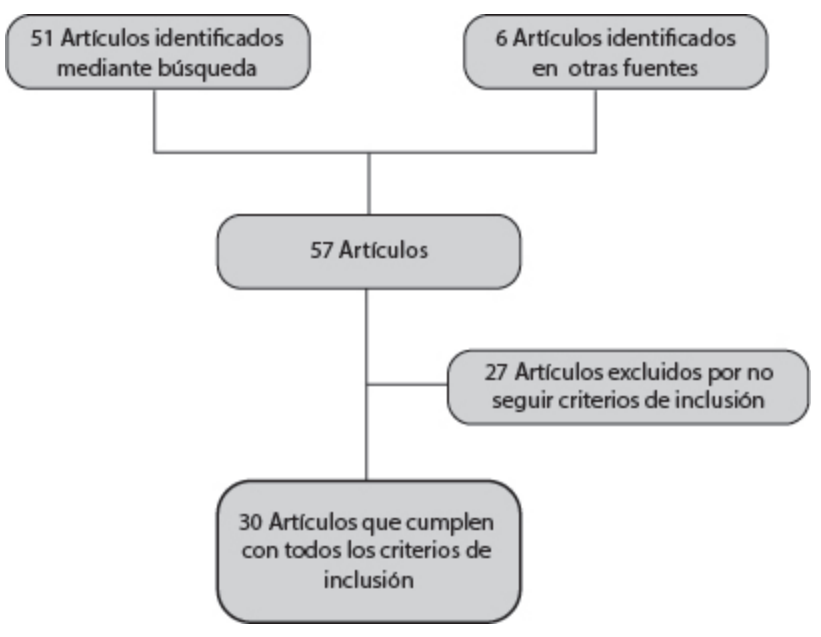

Figura 1. Diagrama de flujo de artículos incluidos y excluidos.

\section{RESULTADOS}

Luego de aplicar criterios de inclusión y exclusión se seleccionaron 32 artículos, entre ellos revisiones sistemáticas y estudios prospectivos y retrospectivos.

El movimiento ortodóncico se basa en una respuesta fisiológica de los tejidos que soportan la estructura dentaria; donde la aplicación prolongada de presión sobre los dientes da como resultado el fenómeno de remodelado de estructuras dentarias y peridentarias, las cuales involucran tanto al ligamento periodontal como al hueso alveolar ${ }^{2,14,17}$.

Específicamente, el proceso inflamatorio que se desarrolla luego de ser aplicado el estímulo mecánico, implica una liberación de mediadores químicos, como histamina, sustancia P, prostaglandinas, entre otros. Esto provoca cambios en el flujo, permeabilidad y calibre de los vasos sanguíneos, lo cual se asocia a períodos de incomodidad relatados por los pacientes, posterior a la aplicación de fuerzas ortodóncicas ${ }^{2}$.

\section{Ciclooxigenasas}

La ciclooxigenasa, también llamada endoperóxido de prostaglandina, es una enzima que permite al cuerpo humano producir mediadores químicos, denominados prostaglandinas, a partir de un ácido graso polinsaturado ubicado en casi todas las membranas celulares del organismo, llamado ácido araquidónico ${ }^{18,19}$.
Existen 3 isoformas de esta enzima:

COX-1: Se encuentra ubicada en todas las células del organismo, expresando constitutivamente en los tejidos. Cumple un rol fundamental en la síntesis de prostanoides, que intervienen en procesos fisiológicos y regula funciones como: protección gastrointestinal, hemostasia vascular, hemodinámica renal y función plaquetaria ${ }^{18,19,20}$.

COX-2: No se encuentra presente normalmente en los tejidos, la célula es estimulada o inducida por agentes como lipopolisacáridos, factores de crecimiento, citoquinas y factores séricos, y regula la síntesis de prostanoides que participan en procesos inflamatorios y no inflamatorios $1^{8,19,20}$.

COX-3: Descubierta recientemente, se encuentra ubicada en el tejido cerebral, la cuál podría ser inhibida por el paracetamol de forma selectiva ${ }^{18,19}$.

\section{Prostaglandinas}

Son sustancias químicas derivadas del metabolismo del ácido araquidónico y forman parte de la familia de prostanoides. Estas actúan como mediadores químicos de la inflamación, interviniendo en los procesos de remodelado óseo inducido por fuerzas mecánicas, aumentando así el movimiento dental en tratamientos ortodóncicos. En altas concentraciones estos mediadores pueden asociarse con un alto riesgo de provocar reabsorción radicular externa ${ }^{2,18,19}$.

Las prostaglandinas (PG) actúan produciendo un aumento del AMPc intracelular y disminuyendo la síntesis de colágeno, además se ha comprobado su capacidad para estimular la reabsorción ósea, especialmente las PG de la serie $\mathrm{E}^{2,18,19}$.

El proceso inflamatorio relacionado con ortodoncia permite el incremento de la permeabilidad de los vasos sanguíneos y estimula la infiltración de linfocitos, monocitos y macrófagos al tejido inflamado, donde las PG son liberadas luego de ser sintetizadas. Estas promueven el remodelado óseo, estimulando e incrementando en número y tamaño las células osteoclásticas del tejido óseo².

Existen diversos tipos de PG, en esta revisión solo se hablará de las más relevantes, las pertenecientes a 
la serie E (E1 y E2). Las PG E1 y PG E 2 son mediadores químicos que juegan un rol fundamental en el proceso de remodelado óseo, porque osteoclastos y osteoblastos durante este proceso aumentan las concentraciones de AMPc y disminuye la síntesis de colágeno, permitiendo el movimiento dentario en tratamientos ortodóncicos. Estas sustancias en altas concentraciones pueden asociarse con el fenómeno de reabsorción radicular, el cual es frecuente en ortodoncia, especialmente en incisivos superiores. Estudios histológicos afirman que se produce un $90 \%$ de rizálisis en piezas dentarias sometidas a tratamiento del especialista, siendo provocado por las fuerzas mecánicas aplicadas durante el tratamiento ortodóncico. La PG $\mathrm{E}_{2}$ es un importante mediador de vasodilatación y aumento de permeabilidad de vasos sanguíneos, por lo que también se encuentra asociada con los períodos de dolor y fiebre ${ }^{2,19,21}$.

El mecanismo del movimiento dental puede encontrarse asociado con la liberación de mediadores químicos de la inflamación, como PG E, las cuales interactúan con células del tejido óseo. Estas aumentan sus niveles al poco tiempo de ser aplicado el estímulo mecánico sobre la estructura dentaria, aumentando sus concentraciones en el ligamento periodontal y el fluido gingival crevicular, por lo tanto, se encuentran en mayores cantidades durante el movimiento dental ${ }^{14}$. Las PG son reconocidas como un potente estimulador de reabsorción de tejido óseo. Se ha observado que estas sustancias químicas aumentan la sensibilidad de los nociceptores involucrados con la respuesta dolorosa, existiendo una variedad de ellas, siendo las más relevantes PG E1 y E ${ }_{2}$, pues contribuyen al movimiento dental ortodóncico y están involucradas en la mediación del dolor ${ }^{1,7,13}$.

\section{Analgésicos antiinflamatorios no esteroidales (AINES)}

A través de los años, la literatura describe diferentes mecanismos para controlar el dolor en ortodoncia, nombrados anteriormente, destacando el uso de analgésicos antiinflamatorios no esteroidales (AINES), siendo este el método comúnmente utilizado durante el tratamiento ortodóncico ${ }^{7}$.

Los AINES presentan propiedades analgésicas, antipiréticas y antiinflamatorias, actúan sobre la biosíntesis del ácido araquidónico, actuando selectivamente sobre la enzima ciclooxigenasa. Por tanto, impiden la síntesis de mediadores químicos como prostaglandinas; siendo estas un importante mediador para permitir el proceso de remodelado óseo y el reclutamiento de osteoclastos y osteoblastos, por ende, permitir el movimiento y desplazamiento dentario durante el tratamiento ortodóncico $^{3,6,7,13,14}$. Estos medicamentos pueden interferir en el movimiento dentario, alterando la reabsorción y aposición de tejido óseo en las zonas de presión y tensión respectivamente, es decir, afectando la actividad de células del hueso alveolar involucradas, debido a la inhibición de la síntesis de prostanoides?

Uno de los AINES mayormente estudiados es el Ibuprofeno, derivado del ácido propiónico. Este medicamento presenta propiedades de analgesia, antipiréticas y antiinflamatorias, y actúa inhibiendo COX competitivamente, tanto COX-1, constitutiva, como COX-2, inductiva $^{22}$. A través de la literatura, se ha observado que este fármaco podría enlentecer el movimiento dental, debido a su acción inhibitoria sobre la síntesis de prostaglandinas, disminuyendo la cantidad de osteoblastos y los osteoclastos involucrados en el movimiento dentario, por tanto, interfiriendo el rango de movimiento ${ }^{7,11}$.

Otro AINES comúnmente utilizado es el paracetamol, también denominado acetaminofeno, con acción principalmente analgésica y antipirética. Presenta una escasa o nula acción antiinflamatoria, pues es un débil inhibidor de prostaglandinas, debido a que su acción ocurre fundamentalmente a nivel del sistema nervioso central, inhibiendo COX-3 en el cerebro y médula espinal. Por lo cual, el grado y velocidad del movimiento dental ortodóncico no se ve alterado significativamente. Es por esto que, no alteraría el remodelado óseo ${ }^{6,11,13,14,22}$.

Así como el paracetamol e ibuprofeno, el naproxeno también pertenece a la familia de AINES. Es un derivado del ácido propiónico, que presenta acción analgésica de mayor duración que el Ibuprofeno. Es un fármaco no selectivo, es decir, genera una inhibición variable de COX1 y COX-2, inhibiendo así la síntesis de prostaglandinas $\mathrm{y}$ afectando el proceso inflamatorio involucrado en el movimiento ortodóncico ${ }^{23}$.

Dentro de esta familia también encontramos el diclofenaco, derivado del ácido fenilacético. Este fármaco posee propiedades analgésicas, antipiréticas y antiinflamatorias. Es un inhibidor de COX no selectivo, suprime la acción de esta enzima, necesaria para la síntesis 
de varios prostanoides, como prostaglandinas y tromboxano A2. Este medicamento retrasa la reparación y crecimiento de tejidos, afectando la cantidad de células encargadas del remodelado óseo. Es por esto, que afecta el movimiento dental ortodóncico, ya que inhibe la síntesis de prostaglandinas relacionadas con el proceso inflamatorio involucrado en dicho tratamiento ${ }^{7,23}$.

Tal como los medicamentos anteriormente nombrados, el ácido acetil salicílico, también denominado aspirina, forma parte de la familia de AINES. Este fármaco tiene propiedades analgésicas y antipiréticas, y su mecanismo de acción es a través de su unión irreversible a la enzima COX. En la literatura se afirma que este medicamento puede prolongar la duración del tratamiento ortodóncico, al igual que el Ibuprofeno. Esto se debe a que ambos fármacos inhiben en mayor medida la producción de prostaglandinas, viéndose reducida la aposición y reabsorción de tejido óseo. Es por esto, que se recomienda evitar dicho medicamento en pacientes sometidos a tratamientos de ortodoncia ${ }^{13,23}$.

Otro medicamento del grupo de AINES, como indometacina y flurbiprofeno son inhibidores selectivos de PG. Ambos fármacos reducen significativamente la cantidad de osteoclastos presentes en el tejido óseo (en un 50\% aproximadamente), por lo cual alteran el curso normal del proceso de remodelado óseo involucrado en tratamientos ortodóncicos. Es por esto que no se recomienda su administración en ortodoncia ${ }^{13}$.

A su vez, el meloxicam, corresponde a un inhibidor relativamente selectivo a la enzima COX 2. Al ser comparado con otros medicamentos como paracetamol e ibuprofeno, este presenta menores efectos adversos a nivel gástrico, siendo entonces el fármaco de elección en el manejo y control del dolor posterior a tratamientos de endodoncia y cirugía oral. En la literatura, se observó que después de dos semanas de administración de este medicamento no se ve afectado el movimiento dental ortodóncico y se cree que es más seguro que otros AINES selectivos ${ }^{8}$.

Un medicamento recientemente estudiado es el etoricoxib. Corresponde a un fármaco de segunda generación, altamente selectivo para la enzima COX-2 con propiedades analgésicas y antiinflamatorias, que posee un efecto inhibitorio dosis-dependiente sobre dicha enzima y vida media de 22 horas (larga) y que ha sido desarrollado para tratamientos de osteoartritis, artritis reumatoide y dolor. Este fármaco al ser un inhibidor de COX-2 tiene la ventaja de disminuir la inflamación en los sitios afectados, convirtiéndolo en un medicamento efectivo y bien tolerado en tratamientos ${ }^{9,24}$.

\section{DISCUSIÓN}

La literatura es extensa y a pesar de la gran cantidad de evidencia científica, los investigadores aún no han logrado definir un estándar de atención para el manejo y control del dolor provocado por los tratamientos ortodóncicos. Es por esta razón, que a continuación se realiza un análisis de la literatura disponible sobre AINES, buscando proponer el medicamento más apropiado para ser utilizado en ortodoncia.

Luego de una búsqueda exhaustiva de la literatura disponible, se encontraron diversos estudios comparativos de fármacos utilizados en tratamientos ortodóncicos, dentro de los cuales se analizó la investigación de Arias et al. ${ }^{13}$ quienes realizaron un ensayo clínico aleatorizado controlado, donde compararon el uso de aspirina, paracetamol e ibuprofeno, relacionándolos con el movimiento dental ortodóncico y analizando sus efectos histológicos en ratas Wistar. Observaron un menor número de osteoclastos en las zonas de presión en ratas tratadas con aspirina e ibuprofeno, en comparación con el grupo control y el grupo tratado con paracetamol. Concluyeron que tanto el ibuprofeno como la aspirina, reducen significativamente las zonas de reabsorción de osteoclastos en las zonas de presión, a diferencia del paracetamol que no lo afecta. Esto puede deberse a que el paracetamol no altera la síntesis de prostaglandinas en el tejido óseo, por lo tanto, no altera el tratamiento ortodóncico, debido a que su acción se concentra en el sistema nervioso central (SNC).

En concordancia con Arias et al. ${ }^{13}$; Salmassian et al. ${ }^{1}$, realizaron un ensayo clínico aleatorizado controlado, donde compararon la eficacia del ibuprofeno, el paracetamol y un placebo, concluyendo que no existen mayores diferencias estadísticas entre ambos AINES para el manejo del dolor, pero recomendaron el paracetamol como el medicamento de elección, dado que presenta pocas reacciones adversas reportadas en la fisiología del movimiento dental y propiedades an- 
tiinflamatorias débiles a nivel periférico, pues su acción concentrada a nivel del SNC ${ }^{7,25}$.

Por otra parte, Polat et al. ${ }^{26}$ hicieron un estudio prospectivo de 150 individuos, donde se comparó AINES como ibuprofeno, paracetamol, naproxeno, flurbiprofeno, aspirina y placebo, administrados una hora antes de la atención y 6 horas después de la instalación de la aparatología ortodóncica, demostrando que el naproxeno presenta el mayor efecto en la disminución de los niveles de dolor. Esto puede deberse a que es un fármaco de larga efectividad, ya que puede mantener sus altas concentraciones en el plasma sanguíneo. $\mathrm{Pa}$ ralelo al estudio anteriormente mencionado, Bartzela et al. ${ }^{25}$ realizaron una revisión sistemática de la literatura el año 2009 sobre los efectos de los medicamentos en la tasa de movimiento ortodóncico, donde afirmaron que el paracetamol tiene un bajo efecto antiinflamatorio por su acción preferentemente sobre sistema nervioso central, no afectando la síntesis de prostaglandinas y por tanto, no alterando el remodelado óseo ni el movimiento dental durante el tratamiento ortodóncico. Debido a que el paracetamol no afecta la velocidad del movimiento dental ortodóncico con dosis terapéuticas, los estudios sugieren que debería ser el analgésico de elección para controlar el dolor asociado con la terapia de ortodoncia.

Por otro lado, Zambrano et al. ${ }^{5}$ encontraron que ciertas investigaciones afirmaban que el paracetamol, usado por sí solo, no es uno de los fármacos que más reduce las molestias causadas por ortodoncia. Por lo cual, realizaron un ensayo clínico aleatorizado controlado en ratas Wistar machos, donde combinaron paracetamol con codeína, obteniendo reducciones significativas en el control del dolor. Postulaban que el paracetamol por sí solo no retarda el movimiento dental pero presenta una baja efectividad analgésica. Ambos medicamentos comparados, actúan directamente sobre el sistema nervioso central y no a nivel periférico.

Otro ensayo clínico aleatorizado controlado, realizado por Kehoe et al. ${ }^{27}$ compararon el efecto del paracetamol, ibuprofeno y misoprostol sobre la síntesis de prostaglandina $\mathrm{E}_{2} \mathrm{y}$ el grado $\mathrm{y}$ tasa de movimiento dental ortodóncico en conejillos de india, indicando que el primero es el analgésico preferido para aliviar el dolor provocado por tratamientos ortodóncicos.
Dicho medicamento es el adecuado porque no altera la respuesta inflamatoria desarrollada en los tejidos, en respuesta a la aplicación de fuerzas mecánicas, y no altera la reabsorción y aposición del tejido óseo, concordando con las investigaciones realizadas por los autores Arias et al..$^{13}$ Salmassian et al. ${ }^{1}$ y Bartzela et al. ${ }^{25}$.

Por otra parte, Saquelli et al. ${ }^{7}$ realizaron un ensayo clínico aleatorizado controlado en conejillos de india, de dos AINES, paracetamol e ibuprofeno, descubriendo que no existe diferencias significativas entre ambos en relación con la percepción del dolor que experimenta el paciente. Ambos AINES estudiados demostraron disminuir los niveles de dolor al segundo día de su administración, por lo cual son altamente efectivos, obteniendo un resultado diferente al resto de los autores y discrepando con sus investigaciones realizadas.

Al igual que otros autores, Angelopoulou et al. ${ }^{28}$ realizaron un meta análisis, donde no encontraron diferencias estadísticamente significativas entre la administración de paracetamol e ibuprofeno para aliviar cuadros de dolor. La literatura explica que el ibuprofeno, en comparación con un placebo, ayuda a aliviar las fases iniciales del dolor, al ser administrado dos y seis horas después de la atención, luego de la intervención del especialista; pero no disminuye significativamente el dolor a las 24 horas, momento en que las molestias alcanzan su pick.

De igual manera, Krasny et al. ${ }^{29}$ afirmaron que el uso de AINES impide el movimiento dental y aumenta el riesgo de reabsorción radicular de piezas dentarias sometidas a tratamiento ortodóncico. Los AINES inhibidores de COX no selectivos presentan desventajas que incluyen inflamación, sangrado gingival y alteraciones de secreción salival. Por lo tanto, consideran el paracetamol como el medicamento de elección, debido a que no ejerce acción sobre el rango de movimiento dental descrito anteriormente, tampoco provocan reabsorción radicular externa, ni efectos adversos gastrointestinales, entre otras reacciones adversas que pueden producirse en la cavidad oral, que son provocados por el uso de otros AINES, como ibuprofeno.

De la misma forma, los investigadores Najafi et al. ${ }^{8}$ realizaron un ensayo clínico aleatorizado controlado, y compararon paracetamol, ibuprofeno y meloxicam 
luego de la colocación de separadores utilizando la Escala Visual Análoga. Los resultados no demostraron diferencias significativas entre la administración preoperatoria del meloxicam y los otros dos medicamentos, posterior a la colocación de los separadores en cuanto al manejo y control del dolor. Esta investigación concluye, al igual que autores anteriores, que el paracetamol puede ser considerado como el AINES más apropiado para el manejo del color, debido a que no provoca reacciones adversas gastrointestinales como perforaciones y úlceras, y porque no afecta el rango de movimiento dental. A su vez, consideran que el meloxicam puede ser recomendado como una buena alternativa para aquellos pacientes a quienes no se les puede administrar otros AINES o paracetamol, esto también debido a su baja toxicidad gastrointestinal.
Los investigadores Sandhu et al. ${ }^{9}$ hicieron un metaanálisis en el año 2016, en el cual afirman que el etoricoxib preoperatorio y postoperatorio es el más efectivo de los AINES en cuanto a sus propiedades analgésicas. Estos descubrimientos concuerdan con otros estudios analizados por estos autores, que reportan que este medicamento es más efectivo para el control del dolor agudo en comparación con otro AINES. Concluyeron que el etoricoxib es el analgésico más efectivo, debido a que sus efectos son dosis-dependientes y presenta una vida media larga.

Como se ha analizado (Tabla 1), múltiples autores apoyan que el uso de AINES no tiene efectos sobre el movimiento dental siempre y cuando sean administrados en bajas dosis por cortos períodos de tiempo. Por lo

Tabla 1. Resumen artículos analizados en discusión

\begin{tabular}{|c|c|c|c|c|}
\hline Autores & $\begin{array}{l}\text { Revista de } \\
\text { Publicación }\end{array}$ & $\begin{array}{c}\text { Año } \\
\text { Publicación }\end{array}$ & $\begin{array}{l}\text { Tamaño de } \\
\text { muestra }\end{array}$ & Conclusión \\
\hline Salmassian et al. & AJODO & 2009 & $\begin{array}{l}60 \text { pacientes dividi- } \\
\text { dos aleatoriamente } \\
\text { en } 3 \text { grupos }\end{array}$ & $\begin{array}{l}\text { Recomendaron el paracetamol como el medicamento de elección, dado } \\
\text { que presenta pocas reacciones adversas reportadas en la fisiología del mo- } \\
\text { vimiento dental y propiedades antiinflamatorias débiles a nivel periférico. }\end{array}$ \\
\hline Arias et al. & AJODO & 2006 & $\begin{array}{l}36 \text { ratas Wistar adul- } \\
\text { tas divididas aleato- } \\
\text { riamente en } 4 \text { grupos }\end{array}$ & $\begin{array}{l}\text { El paracetamol probablemente no altera la regeneración ósea o el movi- } \\
\text { miento dental porque actúa a nivel del sistema nervioso central y no afecta } \\
\text { la secreción periférica de PG. }\end{array}$ \\
\hline Polat et al. & Angle Orthod & 2005 & $\begin{array}{l}150 \text { pacientes di- } \\
\text { vididos aleatoria- } \\
\text { mente en } 6 \text { grupos }\end{array}$ & $\begin{array}{l}\text { Todos los grupos presentaron disminución de dolor, pero el grupo de pa- } \\
\text { cientes con naproxeno sódico presentaron el mayor efecto en la disminu- } \\
\text { ción de los niveles de dolor. }\end{array}$ \\
\hline Bartzela et al. & AJODO & 2009 & $\begin{array}{l}\text { Revisión sistemáti- } \\
\text { ca de } 49 \text { artículos }\end{array}$ & $\begin{array}{l}\text { Debido a que el paracetamol no afecta la velocidad del movimiento dental or- } \\
\text { todóncico con dosis terapéuticas, los estudios sugieren que debería ser el anal- } \\
\text { gésico de elección para controlar el dolor asociado con la terapia de ortodoncia. }\end{array}$ \\
\hline Zambrano et al. & Ciencia UAT & 2008 & $\begin{array}{l}20 \text { ratas Wistar ma- } \\
\text { cho adultas dividi- } \\
\text { das en } 3 \text { grupos }\end{array}$ & $\begin{array}{l}\text { Se deduce que el paracetamol y codeína no inhiben el movimiento den- } \\
\text { tario, debido a que ambos actúan directamente sobre el sistema nervioso } \\
\text { central y no de manera periférica y ayudan a reducir los niveles de dolor. }\end{array}$ \\
\hline Kehoe et al. & Angle Orthod & 1996 & $\begin{array}{l}40 \text { conejillos de indias } \\
\text { divididos en } 3 \text { grupos } \\
\text { aleatoriamente }\end{array}$ & $\begin{array}{l}\text { Paracetamol es el medicamento adecuado porque no altera la respuesta in- } \\
\text { flamatoria desarrollada en los tejidos, en respuesta a la aplicación de fuer- } \\
\text { zas mecánicas, y no altera la reabsorción y aposición del tejido óseo, }\end{array}$ \\
\hline $\begin{array}{l}\text { Angelopoulou } \\
\text { et al. }\end{array}$ & $\begin{array}{l}\text { Orthodontics } \\
\text { \& Craneofacial } \\
\text { Research }\end{array}$ & 2012 & $\begin{array}{l}\text { Metaanálisis de } 7 \\
\text { artículos }\end{array}$ & $\begin{array}{l}\text { El ibuprofeno puede reducir el dolor a las } 6 \mathrm{~h} \text { después del procedimiento } \\
\text { de ortodoncia, mientras que tiene un efecto estadísticamente no significa- } \\
\text { tivo a las } 24 \mathrm{~h} \text {, el tiempo máximo de dolor, después de la colocación de los } \\
\text { separadores o del arco. }\end{array}$ \\
\hline Krasny et al. & $\begin{array}{l}\text { Acta Pol } \\
\text { Pharmac }\end{array}$ & 2013 & $\begin{array}{l}\text { Analgésicos utili- } \\
\text { zados para aliviar } \\
\text { dolor ortodóncico } \\
\text { fueron divididos en } \\
\text { grupos y analizados }\end{array}$ & $\begin{array}{l}\text { La falta de efectos adversos peligrosos proporciona paracetamol con la po- } \\
\text { sición número uno entre los analgésicos utilizados en mujeres embarazadas } \\
\text { y niños pequeños. Además no afecta el rango del movimiento dental ni } \\
\text { aumenta el riesgo de reabsorción de la raíz en los dientes movidos. Y el uso } \\
\text { de Celecoxib a corto plazo durante dos días no afecta el movimiento dental } \\
\text { y puede constituir un tratamiento alternativo para el paracetamol. }\end{array}$ \\
\hline Najafi et al. & $\begin{array}{l}\text { Progress in } \\
\text { Orthodontics }\end{array}$ & 2015 & $\begin{array}{l}321 \text { pacientes di- } \\
\text { vididos aleatoria- } \\
\text { mente en } 3 \text { grupos }\end{array}$ & $\begin{array}{l}\text { El paracetamol se puede considerar como el tratamiento de elección debido } \\
\text { a que no causa úlceras gastrointestinales y no afecta la velocidad del movi- } \\
\text { miento del diente. Teniendo en cuenta la baja toxicidad gastrointestinal, el } \\
\text { meloxicam puede recomendarse como una buena alternativa para aquellos } \\
\text { pacientes que no pueden tomar otros AINES o paracetamol. }\end{array}$ \\
\hline Sandhu et al. & AJODO & 2016 & $\begin{array}{l}\text { Metaanálisis de } 24 \\
\text { artículos }\end{array}$ & $\begin{array}{l}\text { El etoricoxib es el analgésico más efectivo, debido a que sus efectos son } \\
\text { dosis-dependientes y presenta una vida media larga. }\end{array}$ \\
\hline
\end{tabular}


tanto, en pacientes que no presentan condiciones sistémicas relevantes el organismo se encarga de eliminar el medicamento antes de que se produzca el movimiento propiamente dicho. Pero por otra parte, existen estudios que declaran la existencia de disminución o inhibición del movimiento dental ortodóncico como resultado del uso de AINES, debido a su acción sobre la síntesis de prostaglandinas, como el ibuprofeno ${ }^{30}$.

\section{CONCLUSIONES}

Es de suma importancia que el Ortodoncista considere como alternativa terapéutica el uso de AINES para el manejo y control del dolor en pacientes portadores de aparatología fija y complementando el tratamiento ortodóncico y aspirar al éxito.

Según la evidencia científica, aún no se ha definido un estándar de atención para manejar los períodos de incomodidad causados por el tratamiento ortodóncico.

Dentro de la familia de los AINES no se recomienda la administración de ibuprofeno, diclofenaco, naproxeno y aspirina, pues tienen acción sobre las COX, afectando la biosíntesis de prostaglandinas, y por tanto, alterando el remodelado óseo, disminuyendo el rango de movimiento dentario que se busca alcanzar por el especialista.

Se concluyó, que el paracetamol, debido a su acción preferentemente a nivel de SNC, la presencia de pocas reacciones adversas registradas y que no actúa inhibiendo directamente la síntesis de prostaglandinas en los tejidos, se recomienda como medicamento de elección en tratamientos de ortodoncia, pues no altera el curso normal del proceso inflamatorio y movimiento dental.

La presente investigación sugiere que se hagan estudios sobre medicamentos como indometacina, diclofenaco, flurbiprofeno y etoricoxib, debido a que existe escaza evidencia al respecto.

\section{REFERENCIAS}

1. Salmassian R, Oesterle LJ, Shellhart WC, Newman SM. Comparison of the efficacy of ibuprofen and acetami- nophen in controlling pain after orthodontic tooth movement. Am J Orthod Dentofac Orthop. 2009;135(4):51621 [Internet]. American Association of Orthodontists. DOI: 10.1016/j.ajodo.2007.05.020.

2. Peña-Montero CA, Rojas-García AR, Gutiérrez-Rojo JF. Mediadores químicos y el efecto de los analgésicos en el tratamiento de ortodoncia. 2012;1(2):55-61.

3. Patel S, Mcgorray SP, Yezierski R, Fillingim R, Logan H, Wheeler TT. Effects of analgesics on orthodontic pain. Am J Orthod Dentofac Orthop. 2011;139(1):e53-8. [Internet]. American Association of Orthodontists. DOI: 10.1016/j.ajodo.2010.07.017.

4. Polat Ö. Pain and Discomfort After Orthodontic Appointments. Semin Orthod. 2007;13(4):292-300. DOI: 10.1053/j.sodo.2007.08.010

5. Zambrano D, Zamarripa E, Parra R. Acetaminofén con codeína eficiente contra el dolor dentario. Ciencia UAT. 2008;3(2):48-51.

6. Ellison DL. Physiology of Pain. Crit Care Nurs Clin NA. 2017;29(4):397-406. [Internet]. Elsevier Inc; DOI: 10.1016/j.cnc.2017.08.001.

7. Saquelli A, Orellana A, Garzón R. Alternativas de tratamiento para disminuir el dolor de origen ortodóncico. Rev Latinoam Ortod y Odontopediatría. 2010; Disponible en: https://www.ortodoncia.ws/publicaciones/2010/ art-6/.

8. Najafi HZ, Oshagh M, Salehi P, Babanouri N, Torkan $S$. Comparison of the effects of preemptive acetaminophen, ibuprofen, and meloxicam on pain after separator placement: a randomized clinical trial. Prog Orthod. 2015;16(34):3-9. [Internet]. Progress in Orthodontics. DOI: $10.1186 / \mathrm{s} 40510-015-0104-\mathrm{y}$.

9. Sandhu SS, Cheema MS, Khehra HS. Comparative effectiveness of pharmacologic and nonpharmacologic interventions for orthodontic pain relief at peak pain intensity: A Bayesian network meta-analysis. Am J Orthod Dentofac Orthop. 2014;150(1):13-32. [Internet]. American Association of Orthodontists. DOI: 10.1016/j. ajodo.2015.12.025.

10. Ashley PF, Parekh S, Moles DR, Anand P, MacDonald LC. Preoperative analgesics for additional pain relief in children and adolescents having dental treatment. Cochrane Database Syst Rev. 2016(8):Cd008392. DOI: 10.1002/14651858.CD008392.pub3.

11. Bird S, Williams K, Kula K. Preoperative acetaminophen vs ibuprofen for control of pain after orthodon- 
tic separator placement. Am J Orthod Dentofac Orthop. 2007;132(4):504-10. DOI: 10.1016/j.ajodo.2006.11.019.

12. Xiaotinga L, Yin T, Yangxi C. Interventions for pain during fixed orthodontic appliance therapy. Angle Orthod. 2010;80(5):925-32. DOI: 10.2319/010410-10.1.

13. Arias O, Márquez-Orozco MC. Aspirin, acetaminophen, and ibuprofen: Their effects on orthodontic tooth movement. Am J Orthod Dentofac Orthop. 2006;130(3):36470. DOI: 10.1016/j.ajodo.2004.12.027.

14. Vargas P, Piñeiro M, Palomino H, Torres-Quintana M. Factores modificantes del movimiento dentario ortodóncico. Av Odontoestomatol. 2010;26(1):45-53.

15. Krishnan V, Davidovitch Z. The effect of drugs on orthodontic tooth movement. Orthod Craniofac Res. 2006;9:163-71. DOI: 10.1111/j.1601-6343.2006.00372.x.

16. Primo J. Niveles de evidencia y grados de recomendación (I/II). Enferm Inflamatoria Intest al día. 2003;2:2001-4.

17. Kyrkanides S, O’Banion MK, Subtelny JD. Nonsteroidal anti-inflammatory drugs in orthodontic tooth movement: Metalloproteinase activity and collagen synthesis by endothelial cells. Am J Orthod Dentofac Orthop. 2000;118(2):203-9. DOI: 10.1067/mod.2000.105872

18. Fernández Duharte J, Zapata Blanco E, Santiesteban Sauqué X, Lescay Bell O, Rosell Torres L. Uso y abuso de las prostaglandinas. MEDISAN. 19(1):113-121. [Internet]. 2015 Ene [citado 2018 Ene 15]. Disponible en: http://scielo.sld.cu/scielo.php?script=sci_arttext\&pi$\mathrm{d}=$ S1029-30192015000100015\&lng=es.

19. Gálvez Lima Á. Efecto antiinflamatorios de los ácidos grasos omega - 3. Scientifica. 2007 Sep. 5(5):43-49. [revista en la Internet]. [citado 2018 Ene 15]. Disponible en: http://www.revistasbolivianas.org.bo/scielo.php?scrip$\mathrm{t}=$ sci_arttext\&pid=S1813-00542007000200010\&lng=es.

20. García JA, Gómez-Reino J. Fisiopatología de la ciclooxigenasa-1 y ciclooxigenasa-2. Rev Española de Reumatología. 2000;27:33-5.

21. Vaquero P, B P, E L, Santiago A GF. Reabsorción radicular durante el tratamiento ortodóncico: causas y recomendaciones de actuación. Cient Dent. 2011;8(1):61-70.
22. Bradley RL, Ellis PE, Thomas P, Bellis H, Ireland AJ, Sandy JR. A randomized clinical trial comparing the efficacy of ibuprofen and paracetamol in the control of orthodontic pain. Am J Orthod Dentofac Orthop. 2007;132(4):511-7. DOI: 10.1016/j.ajodo.2006.12.009

23. Batlouni M. Antiinflamatorios no esteroides: efectos cardiovasculares, cerebrovasculares y renales. Arq. Bras. Cardiol. 2010 Apr;94(4):556-563. [Internet]. [cited 2017 Dec 26]. Available from: http://www.scielo.br/scielo.php?script $=$ sci_arttext\&pid=S0066-782X2010000400019\&ln$\mathrm{g}=\mathrm{en}$.

24. Behal N, Singh Kanwar S, Sharma P, Sanyal SN. Effect of non-steroidal anti-inflammatory drug etoricoxib on the hematological parameters and enzymes of colon and kidney. Nutr. Hosp. 2009 Jun;24(3):326332 [Internet]. [citado 2018 Ene 30]. Disponible en: http://scielo.isciii.es/scielo.php?script=sci_arttext\&pi$\mathrm{d}=$ S0212-16112009000300010\&lng=es.

25. Bartzela, T. Turp, J. Motschall, E. Maltha, J. Medication effects on the rate of orthodontic tooth movement: A systematic literature review. American Journal of Orthodontics. 2009. DOI: 10.1016/j.ajodo.2008.08.016.

26. PolatO,KaramanA.PainControlDuringFixedOrthodontic Appliance Therapy. Angle Orthod. 2005;75:210-215. DOI: 10.1043/0003-3219(2005)075<0210:PCDFOA > 2.0.CO;2.

27. Kehoe MJ, Cohoen SM, Zarrinia K, Cowan A. The effect of acetaminophen, ibuprofen and misoprostolonprostaglandin E2 synthesis and the degree and rate of orthodontic tooth movement. Angle Orthod. 1996;66:339-50. DOI: 10.1043/0003-3219(1996)066<0339:TEOAIA >2.3.CO;2.

28. Angelopoulou MV, Vlachou V, Halazonetis DJ. Pharmacological management of pain during orthodontic treatment: A meta-analysis. Orthod. Craniofac. Res. 2012;15(2):7183. DOI: 10.1111/j.1601-6343.2012.01542.x.

29. Krasny M, Zadurska M, Cessak G, Fiedor P. Analysis of effect of NSAID's drugs on teeth and oral tissues during orthodontic treatment. Report based on literature. Acta Pol. Pharm. 2013;70(3):573-577.

30. Possi A, Gallelli L. Pain management for dentists: The role of Ibuprofen. Annali di Stomatologia. 2011;11(Supply 3-4):3-24. 